\title{
Performance Evaluation \& Operation of DSP Control UPS System
}

\author{
S. M. Sayedur Rahman, Diponkar Paul*, Md. Masud Rana \\ City University, Dhaka, Bangladesh \\ Email: *dipo0001@ntu.edu.sg
}

How to cite this paper: Rahman, S.M.S., Paul, D. and Rana, M.M. (2020) Performance Evaluation \& Operation of DSP Control UPS System. Engineering, 12, 666-681.

https://doi.org/10.4236/eng.2020.129047

Received: June 15, 2020

Accepted: September 26, 2020

Published: September 29, 2020

Copyright (๑) 2020 by author(s) and Scientific Research Publishing Inc. This work is licensed under the Creative Commons Attribution International License (CC BY 4.0).

http://creativecommons.org/licenses/by/4.0/

(c) (i) Open Access

\begin{abstract}
An uninterruptible power supply (UPS) is a power conditioner that provides emergency power to a load when the supply power fails. In on-line UPS, the load is always connected to the inverter through the UPS static switch. Specialized expertise was required for collection, analysis, and dissemination of technical information relating to high quality uninterruptible power systems. Inherent in these responsibilities is the coordination of technical and contractual matters relating to the procurement and installation of uninterruptible power supply (UPS) systems which were required to support these facilities. The proposed circuit incorporates an AC/DC \& DC \& AC converter, which provided power factor correction and to assure feedback control. In our project when the $\mathrm{AC}$ main is available, the rectifier circuit will supply the power to the inverter as well as to the battery and battery will be charged. If the supply power fails suddenly, the battery will supply power to the inverter without any interruption and delay. In the constant current charging technique, a digital charger was designed and controlled through PIC12F675 microcontroller. In the realized system, The PIC16C73B was used as microcontroller. It was used to generate sinusoidal PWM (Pulse Width Modulation) signals that are connected to drive $\mathrm{n}$-channel MOSFETs in inverter to assure feedback control and display units. The power circuit of the proposed UPS system was presented and analyzed. Circuit models were derived and a UPS digital control system using a digital signal processing (DSP) with microcontroller has been developed. The uninterruptible Power Supplies (UPS) are widely used by power supply during interruption of regular power supply due to load shedding, power failure, power fluctuations including telecommunication systems, medical systems, industrial control systems etc.
\end{abstract}

\section{Keywords}

PWM, Inverter, MTBF, ADC, PIC 


\section{Introduction}

Recently, UPSs for server rooms or line factories have been actively researched. An uninterruptible power supply is an electrical apparatus that provides emergency power to load when input source, typically the main source power fails. It provides near instantaneous protection from input power interruptions by supplying energy stored in batteries. Generally, configurations of the UPSs are divided into a standby-type and an online-type. The standby-type UPS has an advantage that when the grid is in normal state, load current flows only through AC switches. Therefore, power loss in the normal state is very small. However, when the grid failure occurs, the detection of the voltage drop takes several milliseconds and the load voltage is interrupted during the detection period. On the other hand, the online-type provides uninterrupted power even when the grid suddenly fails because the online-type UPS provides power by batteries continuously. The on-line UPS provides a conditioned output voltage when the power is on and charges the battery through the battery charger. The control circuits of UPS automatically switch over to the inverter and supply power from the batteries during power interruption/failure. The change over from mains to the battery and back to the mains supply is done automatically by the control circuits. The modern UPS employs MOSFET based inverter and pulse width modulators techniques and static switches. However, this operation requires PWM control which generates switching loss even when the grid is in the normal state. In order to reduce this switching loss, multilevel converters have been proposed for UPS applications. In multilevel converters, by applying devices with low voltage rating, the switching loss can be reduced. However, the PWM method is still necessary to control the input current and the output voltage into the sinusoidal waveform. Therefore, the reduction of the switching loss at stable grid condition is limited due to which the switching frequency of the rectifier part is only six times of the grid frequency has been considered. However, the inverter part is still operated with PWM method, which results in high switching loss. In this paper, the converter which is constructed by a rectifier and an inverter is proposed. The proposed converter significantly reduces the switching loss because the switching frequencies in both the rectifier and the inverter are reduced to six times of the grid frequency. The characteristics of the proposed converter are as follows firstly, when the grid voltage fluctuates, the rectifier in the proposed converter operates in boost mode with PWM method in order to maintain the constant output voltage. Secondly, when the failures of the power grid occur, the load power can be supplied from batteries. Furthermore, the voltage interruption is completely avoided because the DC-link capacitor energy maintains constant output voltage. In other words, this proposed converter not only reduces the switching loss in the stable operation of the grid but also compensates for the grid failures. Therefore, the proposed converter is suitable for the online-type UPS applications [1]. Furthermore, the proposed converter is expected to have high reliability and long lifetime, because instead of electrolytic capacitors, 
the film capacitors can be applied at DC link. However, there is a problem in the proposed method, which is distortion of the input current. The distortion of the input current has two causes; surge offset voltages by the inappropriate commutation of the inverter and discontinuity of the input currents by the improper design of the input LC filter. Therefore, in this paper, in order to suppress the surge voltage and eliminate the discontinuity of the input current, an improved switching method and LC filter design method are proposed. In order to eliminate the surge voltage and the distortion of the input current, a current commutation strategy which uses an overlapped switching in order to ensure the current path is applied. Besides, in order to eliminate the discontinuous current, the LC filter is designed in the condition that the input phase angle is maintained above 30 degree. This paper is organized as follows: first, the control method of the proposed converter is explained and the basic operation is confirmed; second, the problems are described and analyzed. Third, the improved switching method and the LC filter design method of the proposed circuit are explained. Real time PWM control of the inverter output voltages provides the ability to dynamically adapt to changing load conditions. This paper presents real time digital signal processing (DSP) control of UPS systems supplying nonlinear loads to continuously provide modulating sinusoidal load voltages. The implementation of the proposed controllers usually involves measurements of the output voltage and either the capacitor current or the inductor current, and in some cases, they even require measurements of the current load with the idea of attenuating the effect of disturbances in the load. The capacitor voltage is introduced in those controllers in a second voltage loop to alleviate imperfections in the response due to parameter uncertainties and load disturbances as well. Adaptive refinements have also been added to the controller to cope with parametric uncertainties. By using the frequency domain descriptions of some unknown signals (disturbance), the solution presented here is able to perform precise voltage tracking (even with nonlinear loads). It reduces the effects of unbalance and harmonic distortion, which is similar to other frequency-domain techniques, such as synchronous frame harmonic control [2]. For such purpose, the system dynamics were modeled using stationary frame quantities and the load currents. Sequence components, i.e., positive and negative, are considered so that the unbalanced case can be treated. The proposed controller realizes a partial inversion of the system and adds the needed damping. The resulting system contains a disturbance term due to uncertainties in the system parameters, which is addressed via adaptation. Due to the complexity of this controller, a simple rotational transformation is proposed so that the computation complexity can be significantly reduced. Similar to other frequency-domain techniques, a group of selected harmonics is taken into account for parameter adaptation and voltage regulation, and thus the proposed approach can be classified as selective, since only a selected set of harmonics is targeted for compensation. The resulting scheme is directly connected to the previous work where the inductor current is used instead. Which include a bank of resonant filters as the main har- 
monic compensation element and were derived following. The solution proposed here is based on a new more rigorous theoretical framework following nonlinear control design techniques and based on the frequency-domain representation of the disturbances. The advantages of DSP control and discussed and a DSP controlled UPS inverter and harmonic conditioning system is described with the performance verified on a 100 VA system. Finally, the proposed control scheme has been implemented and tested in a $100 \mathrm{VA}$ single phase inverter. Finally, the validity of the proposed methods is confirmed by experiment. Prior to the mid 1960's, the Navy's method of providing uninterruptible or no-break ac electrical energy for critical loads consisted of a motor-generator set with a large flywheel. In the event of prime power loss, the generator maintained critical load power for a very short period of time which was dependent on flywheel size and various other mechanical factors. As a result of the rapid development of semi-conductor technology, solid state UPS systems were introduced and gained wide acceptance in the mid 1960's. Since that time, improvements in the "state-of-the-art" of UPS have occurred rapidly. One of the most important of these improvements has been the reduction in UPS system failures. Advances in design and system configuration have steadily improved the efficiency \& Navy procured UPS systems. For example, early systems depended on the UPS as the only power source, therefore, multi-redundant modules were required as insurance against the infrequent failure of one of the modules. These systems, purchased in 1966 and 1969, were expensive and efficient. A major step toward a more economically feasible UPS system was the advent of the static bypass switch in the early 1970's. This static bypass switch allows an uninterrupted transfer to commercial power in the event of an UPS failure, thus the non-redundant system was born. As reflected in the 1973 procurement, the non-redundant system was a low cost and an energy efficient alternative. The cost per KVA of a UPS was further reduced as a result of larger sized UPS modules and improved reliability of the UPS battery system. These three factors allowed a reduction in modules per system and the elimination of redundant batteries as demonstrated by the system. In the Navy's continuing effort to keep abreast of the "state-of-the-art" in solid state UPS, earlier problems with poor reliability and efficiency were effectively resolved during four "generations" of development, resulting in a fourth generation system which is $85 \%$ to $90 \%$ efficient and highly reliable. The non-redundant UPS consists of one UPS module with a static bypass circuit and battery. A UPS module is the static power conversion portion of the UPS system and consists of a rectifier, an inverter, and associated controls along with synchronizing, protective and auxiliary devices. Upon failure of the UPS module, the static bypass circuit automatically transfers the critical load to the primary standby or emergency source without any interruption to the critical technical load, the parallel redundant UPS consists of two or more modules, system control cabinet and a common battery. The modules operate in parallel and each is capable of supplying the rated critical technical load upon failure of any one module. A static interrupter will disconnect a failed module 
from the system output bus as a result of a detected fault without an interruption to the critical technical load, the "cold" standby redundant UPS consists of two independent non-redundant UPS modules with a common battery. One of the non-redundant UPS operates on-line and the other off-line. Should the operating UPS fail; the static bypass switch will automatically transfer the critical technical load to the primary, standby or emergency source without interruption to the load. The second module is then manually energized and placed in the bypass mode of operation. To transfer the critical technical load, the external make-before-break non-automatic circuit breakers are operated to place the load on the second UPS bypass, thereafter that module's static switch is activated to allow the module to supply the critical load. Looking toward the future, the most significant improvement in UPS efficiency is expected to be the "hot" standby non-redundant UPS. An efficiency improvement of $6 \%-8 \%$ in the "hot" standby non-redundant systems and $1 \%-3 \%$ in frequency conversion type redundant systems can be achieved. From this reliability study, the published MTBF of UPS module may not be the most significant factor in determining whether a "load sharing" parallel redundant UPS is more or less reliable than a "cold" standby redundant configuration. Therefore the probability exists that one of many possible human errors will cause a system failure and thereby become the major consideration. It is logical that this probability will be greater in the load-sharing configuration since the UPS modules are not completely independent. In comparing the MTBF of these different configurations, the existence of single point failures is a key issue. Unfortunately, it is very difficult to prove just where these single point failures actually do occur. Most UPS manufacturers describe their "load sharing" parallel redundant systems as containing independent modules such that if one module fails the others will asset the critical load while the failed module is being repaired. The degree of independence of each module of the system is often misinterpreted. Load sharing systems have a significant amount of common control logic that enables parallel operation of the modules. Designing-in redundancy or designing-out single point failures can significantly improve the MTBF of an UPS system. The UPS manufacturer therefore attempts to eliminate all single point failures which would interrupt power to the critical load. However, in the "load sharing" parallel configuration there are circumstances which are beyond the manufacturers' control and limit the extent to which these failure points can be isolated. This may cause the subsequent failure of both modules and loss of power to the critical load [3]. Any of the above may represent single point failures in a "load sharing" parallel UPS configuration. When these kinds of failure modes are included in the MTBF consideration, the "cold" standby redundant UPS with static bypass will have a better MTBF than a load-sharing configuration.

\section{Methodology}

In our DSP control UPS system, the AC voltage applied to the step down transformer. $220 \mathrm{~V}$ AC Convert to the $18 \mathrm{~V} \mathrm{AC}$. An uncontrolled rectifier converts 
AC voltage into DC voltage. Then $14.5 \mathrm{~V}$ applied to the static switch. And at that moment, the charging controller distributes the charge. Both lines are connected to static switch through diode. Then inverter converts the voltage DC to AC. The output voltage of inverter is $12 \mathrm{~V} \mathrm{AC}$. Then we applied the $12 \mathrm{~V}$ AC to a step-up transformer. Then we get $220 \mathrm{~V}-230 \mathrm{~V}$ AC without any noise.

$220 \mathrm{AC}$ voltages are applied to the step down transformer of $0-12 \mathrm{~V} / 1 \mathrm{~A}$. The output of $12 \mathrm{~V}$ ac is converted into $12 \mathrm{~V}$ dc through rectifier as shown in the block diagram of DSP control UPS system in Figure 1. Rectifier with filter capacitor converts AC into $12 \mathrm{~V}$ DC. The capacitors of $1100 \mu \mathrm{F}, 50 \mathrm{~V}$ are used to control the heavy current which may damage the MOSFET switch. The switching frequency of MOSFET switch is $8 \mathrm{kHz}$. Here we used bridge rectifier for removing circuit complexity. The turn on time and turn off time of MOSFET controlled through the isolated driver which may also regulate the voltage. The turn on time of MOSFET-IRFZ44N will be different as for constant current charging. Hence, duty ratio will also be different. The inductor used is of toroidal type. The variable DC is fed to the battery through the resistors of $1 \mathrm{k} \Omega$ and $10 \mathrm{k} \Omega$. The voltage samples are taken between $1 \mathrm{k} \Omega$ and $10 \mathrm{k} \Omega$ resistor. Also other voltage sample is taken across the battery. The voltage samples are given to the PIC 12F675 microcontroller for comparing purpose for constant current charging of battery. When the voltage sample across the battery is less than $14.5 \mathrm{~V}$ then the battery will be charged, and when the voltage sample across the battery is more than 14.5 then stop charging. The special features of PIC12F675 are analog to digital converter, TTL $=$ TTL input buffer, $\mathrm{ST}=\mathrm{Schmitt}$ Trigger input buffer. The operating voltage of pic12F675 is $2 \mathrm{~V}$ to $5.5 \mathrm{~V}$ and Temperature Range (C)-40 to 125 . So, for operating voltage of microcontroller, we used voltage regulator 78L05. We connect the positive terminal through a diode $1 \mathrm{~N} 4007$ and $22 \Omega$ resistor and connect the voltage regulator input terminal which is pin 3 . Here we use N-channel MOSFET (metal-oxide semiconductor field-effect transistor) because the MOSFET is a voltage controlled device. The MOSFET has "gate", "Drain" and "Source" terminals. By applying voltage at the gate, it generates an electrical field to control the current flow through the channel between drain

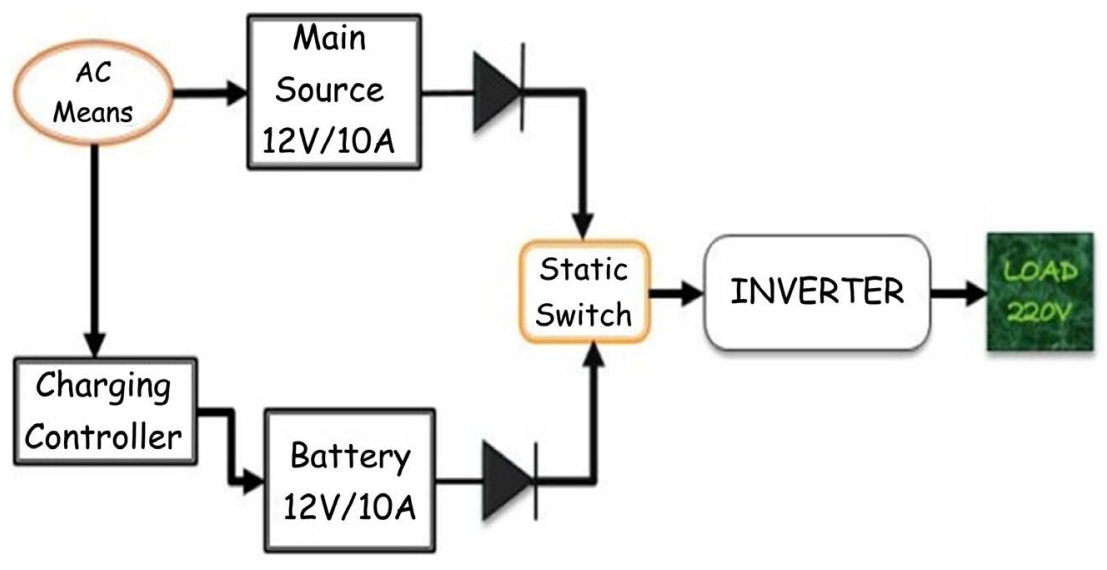

Figure 1. Block diagram of DSP control UPS system. 
and source, and there is no current flow from the gate into the MOSFET. A MOSFET may be thought of as a variable resistor, where the Gate-Source voltage difference can control the Drain-Source Resistance. When there is no applying voltage between the Gate-Source, the Drain-Source resistance is very high, which is almost like an open circuit, so no current may flow through the Drain-Source. When Gate-Source potential difference is applied, the Drain-Source resistance is reduced, and there will be current flowing through Drain-Source, which is now a closed circuit [4]. MOSFETs were chosen for use in this project due to its fast switching rate and ruggedness. The parameters for PIC initialization, then initialization ADC. Then decision making port take decision. If the voltage is greater than $14.5 \mathrm{~V}$ then stop charging and return to PIC initialization I/O. If the charging voltage less than $14.5 \mathrm{~V}$ then keep charging. In our charging unit, we used here $220 \mathrm{~V}$ to $12 \times 2 \mathrm{~V}, 1 \mathrm{~A}$ step-down transformer. Its rated power $24 \mathrm{VA}$. We applied the voltage for regulated DC current.

Figure 2 shows the flowchart consideration for PIC programming. We charged the battery by using this transformer. When the means power available the charging transformer supply the power to rectifier circuit for pure DC then battery will be charged. Rectification is the conversion of alternating current to direct current. Rectification is performed by a diode that allows current to flow in one direction but not in the opposite direction. Direct current that has only been rectified, however, has various changes in voltage (ripples) lingering from the alternating current. Capacitors are used to smooth the current and make it even. In many power supply circuits, the bridge rectifier is used. The bridge rectifier produces almost double the output voltage as a full wave center-tapped

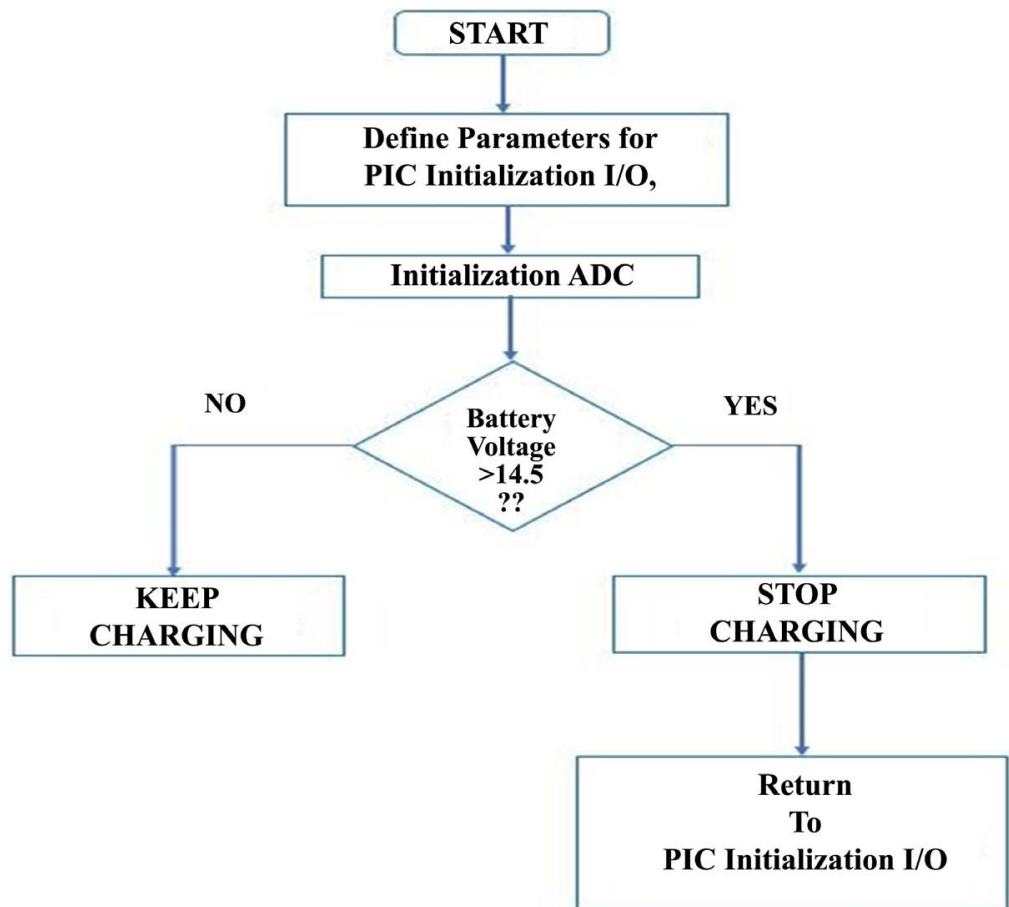

Figure 2. Charging flowchart for PIC programming. 
transformer rectifier using the same secondary voltage. The advantage of using this circuit is that no center-tapped transformer is required [5]. During the positive half cycle, both D3 and D1 are forward biased. At the same time, both D2 and D4 are reverse biased. Note the direction of current flow through the load during the negative half cycle D2 and D4 are forward biased and D1 and D3 are reverse biased. Again note that current through the load is in the same direction although the secondary winding polarity has reversed. In all UPS systems, main goal is to generate ideal output voltage for every kind of load and temporary case. A $50 \mathrm{~Hz}$ sinusoidal output is generally necessary in the UPS systems. Modern inverters work with PWM strategy. Load impedance in the system can be $0 \%$ - $150 \%$ of the nominal load values and also it can be resistive, inductive or capacitive. The feedback is used to assure output voltage at the desired value. Adjusting the output voltage as depends on control strategy is the most important features of the system. The transformer TR3 steps down the mains voltage (220 $\mathrm{V})$ to $18 \mathrm{~V}, 7 \mathrm{~A} \mathrm{AC}$ and then the bridge BR1 rectifies it. The rectified signal is smoothed by the capacitor $\mathrm{C}$. Then connect the line into the static switch. When fault occurs then battery will supply the power to the inverter through 10 $\mathrm{K}, 12 \mathrm{~K}$ and a $10 \mathrm{~K}$ variable resistor. In normal mode, AC mains supply the power to the inverter. The fuse (16 A) acts as a mini circuit breaker for protection against short circuits, before connect the inverter we use this fuse. The block diagram shows input section or source section. From the static transfer switch, the voltage reached to the inverter part of the circuit. The inverter does not produce any power, the power is provided by the DC source. A power inverter, or inverter, is an electronic device or circuitry that changes direct current (DC) to alternating current (AC). The input voltage, output voltage and frequency and overall power handling depend on the design of the specific device or circuit. A power inverter can be entirely electronic or may be a combination of mechanical effects and electronic circuitry. An inverter can produce a square wave, modified sine wave, pulsed sine wave, pulse width modulated wave (PWM) or sine wave depending on circuit design. There are two basic designs for producing household plug-in voltage from a lower-voltage DC source, the first of which boost converter is used to produce a higher-voltage $\mathrm{DC}$ and then converts to AC. The second method converts DC to AC at battery level and uses a line-frequency transformer to create the output voltage. A typical power inverter device or circuit requires a relatively stable DC power source capable of supplying enough current for the intended power demands of the system. The input voltage depends on the design and purpose of the inverter. The input voltage of the inverter is maximum $15 \mathrm{~V} \mathrm{DC}$. The figure shows the block diagram of inverter switching that is DC to AC changing system. For the switch S1 and S2 we use MOSFET. The two switches do not close at a time. When S1 closes then S2 opens. The push-pull configuration of inverter is used for designing of inverter for On-Line UPS. For switching operation, MOSFET Switches IRFZ44N are used. For that MOSFET switches MOSFET driver IRFZ44N is used. The output from pin 15 and 16 as PWM waveforms are fed to the PIC16C73B through the 
transistor BC557, resistor and capacitor $2200 \mu$ respectively. From the static transfer switch at first the current reached to the pin-1 of the PIC16C73B microcontroller.PIC16C73B is a most important microcontroller cheap. It has 28 pin. Pin-1 (MCLR/VPP) is directly connected to the static Transfer switch through a resistor $\mathrm{R}_{16}$ and Pin-2 (RAD/AN0) works as feedback operation. Pin-23, 24, 25, 26, 27, and 28 is connected to the LCD1 pin-4, 6, 12, 13, and 14. It helps us to measure the input voltage and output voltage of our project correctly. The pin-16 is connected to the transistor BC547 through resistor R8. BC547 is an NPN bi-polar junction transistor. A transistor, stands for transfer of resistance, is commonly used to amplify current. A small current at its base controls a larger current at collector \& emitter terminals. BC547 is mainly used for amplification and switching purposes. It has a maximum current gain of 800 . The transistor terminals require a fixed DC voltage to operate in the desired region of its characteristic curves. This is known as the biasing. For applications, the transistor is biased such that it is partly on for all input conditions. The input signal at base is amplified and taken at the emitter. BC547 is used in common emitter configuration for amplifiers. The voltage divider is the commonly used biasing mode. For switching applications, transistor is biased so that it remains fully on if there is a signal at its base. In the absence of base signal, it gets completely off. A small signal NPN transistor used for general purpose audio amplifiers and switching. Then it connects to the another transistor BC 557. It is a transistor used in IR TOGGLE SWITCH. It had been pursuing a Field Effect Transistor (FET) based on copper oxide when they stumbled upon a very different amplifying effect produced by closely-spaced metal contacts touching Germanium semiconductor. Its collector is connected to the MOSFET. We try to use MOSFET to turn the LED on. The source pin of your MOSFET is the output to your LED. But its gate must be $10 \mathrm{~V}$ higher than the source for it to fully turn on. You do not have $+12 \mathrm{~V}$ or higher pulses for the gate The IRFZ44N needs 10 $\mathrm{V}$ pulses at its gate, not just $4 \mathrm{~V}$. A logic-level MOSFE needs $4.5 \mathrm{~V}$. Then the source pin is connected to $0 \mathrm{~V}$ and the LED and its current-limiting resistor is connected between the drain pin and $+10 \mathrm{~V}$. The LED voltage drop is about 1 to $2 \mathrm{~V}$, depending on the LED type, power, etc. Therefore there will be about 8 to 9 $\mathrm{V}$ between the MOSFET drain and source. Draw a vertical line at $8.5 \mathrm{~V}$ and you will see that the line intersects the characteristics at $4.5 \mathrm{~V}$ gate-source if the LED takes $10 \mathrm{~mA}, 5 \mathrm{~V}$ if the LED needs $20 \mathrm{~mA}$, and so on. So, this is the gate-source voltage the MOSFET needs to turn on the LED. Add to this voltage the voltage drop on the LED and you will come up with the minimum gate level in order for the LED to function. At $100 \mathrm{~mA}$, the transistor needs $8 \mathrm{~V}$ gate-source voltage. These pulses are referred to ground. There is connected to PIN no. 17; another same circuit as like as PIN no 16. The circuit from pin 16 and 17 is connected to the step up transformer of value 7.5-0-7.5:250 V. The transformer is step up the voltages from $15 \mathrm{~V}$ to $250 \mathrm{~V}$ AC. That is directly connected to the load. There is also a feedback circuit. The microcontroller is also operated in charge of the synchronization control of all modules of this UPS system as well as the inverter 
feedback control. The line voltage zero-crossing signal is captured by the microcontroller to synchronize the input voltage with the output Voltage. The results of the stability analysis show that a control scheme which employs the filter capacitor current in an inner feedback loop and the load voltage in an outer voltage control loop results in successful operation of the online UPS system. The general noise like as harmonic noise, frequency distortion and electronic noise are removed and resolved. The parameters for PIC initialization, LCD, Timer, ADC and initialization I/P. After that read input voltage. Then decisions making port take decisions. If the input voltage is greater than $10.5 \mathrm{~V}$ then start timer. After that start oscillator and setting clock width. If the input voltage is less than $10.5 \mathrm{~V}$ then stopping timer, stopping oscillator and setting alarm. Then return to the portion read $\mathrm{I} / \mathrm{P}$ voltage. Then read output feedback voltage. If the output voltage is greater than $230 \mathrm{~V}$ then decreases clock width. If not check the output voltage again. If the output voltage is greater than $220 \mathrm{~V}$ then increases clock width and return to the portion read I/P voltage. And if not direct return to the portion read $\mathrm{I} / \mathrm{P}$ voltage. If the input voltage is less than $10.5 \mathrm{~V}$ then stop timer, stop oscillator and set alarm. Then return to the portion read I/P voltage. And this process will be continued. If the output voltage is greater than $230 \mathrm{~V}$ then decreases clock width. If not check the output voltage again. If the output voltage is greater than $220 \mathrm{~V}$ then increases clock width and return to the portion read $\mathrm{I} / \mathrm{P}$ voltage. And if not direct return to the portion read $\mathrm{I} / \mathrm{P}$ voltage.

The hardware set up of Proposed Topology is shown in Figure 3. If the input voltage is less than $10.5 \mathrm{~V}$ then stop timer, stop oscillator and set alarm. Then return to the portion read I/P voltage. And this process will be continued. The output inverter is implemented by two MOSFET transistors in half bridge topology. The inverter is fully digitally controlled and generates a pure sine wave voltage. The sine waveform is generated using the pulse-width modulation technique. The sine reference is stored in a look-up table. The table values are periodically taken from the table, and then multiplied by the required amplitude. The resulting value gives the duty cycle of the PWM output [6]. The pointer to the table is incremented by a value, which corresponds to the desired output frequency. All values over one period give sinusoidal modulated square wave output. If such a signal passes through an LC filter, the pure sine wave voltage is generated on the inverter output.

The inverter simulation is carried out in Figure 4. When an UPS equipment problem occurs, the load is automatically transferred by the static switch bypass to an alternate power source to prevent power interruption to the loads. The static switch is also useful in clearing load faults downstream of the UPS. The static switch will transfer to the alternate power source on a setting of 110 to 125 percent of rated load. Without this feature, the inverter would be driven to current limit on a fault.

Figure 5 shows the result on LCD LM016L at off stage. As this inverter is a pure sine wave harmonics of high frequency have been removed altogether and when it's used with a capacitive load the impedance is as calculated. The problem 


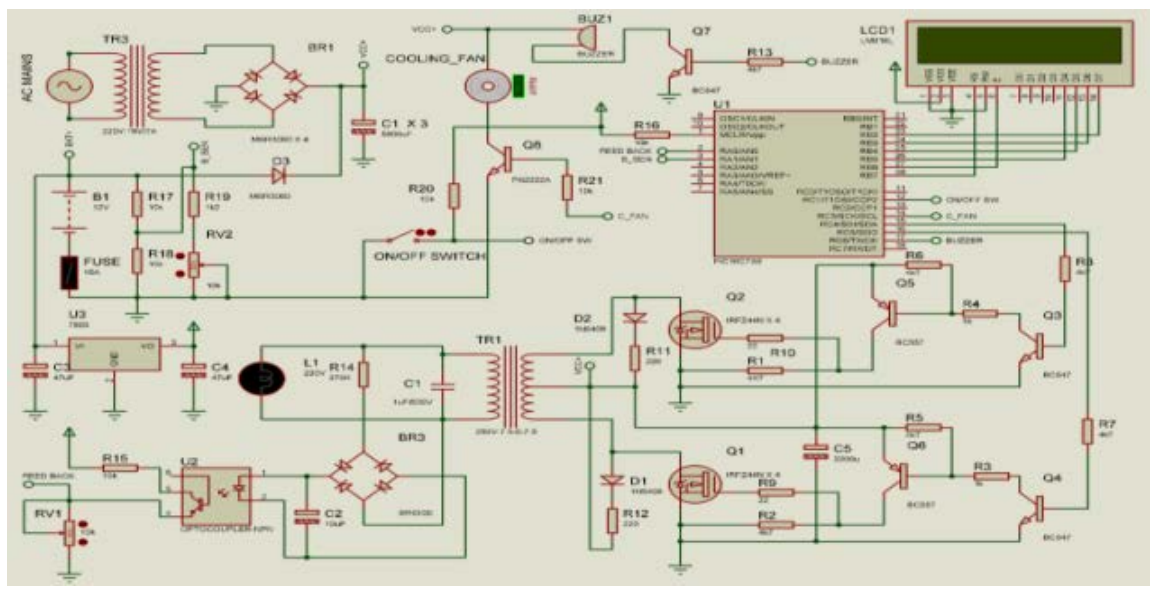

Figure 3. Overall system of ONLINE UPS.

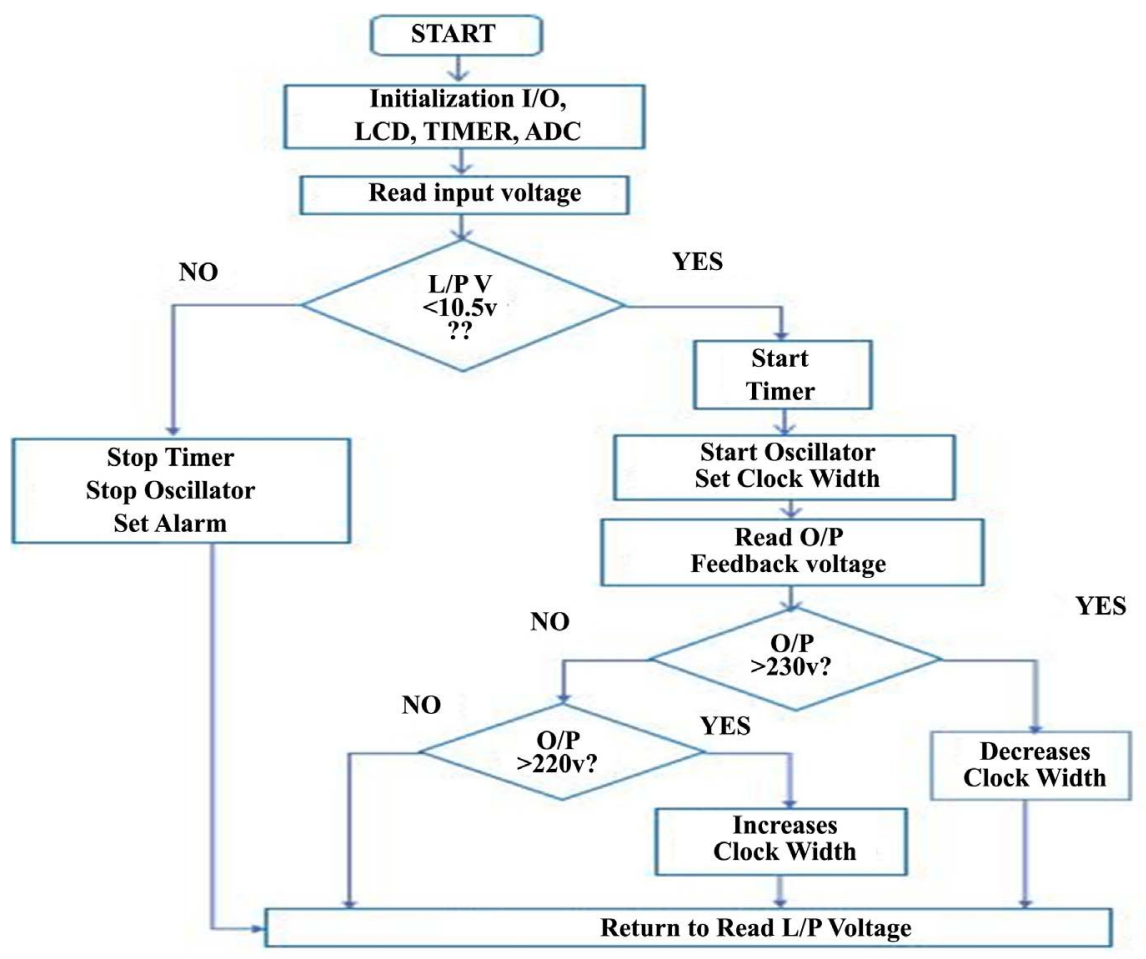

Figure 4. Flowchart of inverter for PIC programming.

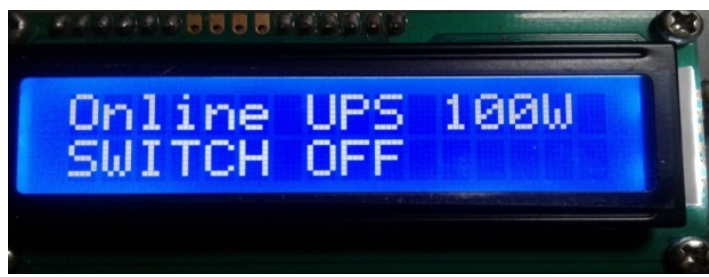

Figure 5. Result on LCD LM016L at off stage.

with other types of inverter is that due to high frequency harmonics the capacitive impedance is lowered hence much current is drawn from the inverter which may exceed the rated current. Rise in temperature is brought by high frequency 
switching of MOSFET which dramatically decreases the power output the inverter. In order to maintain a low temperature all MOSFET and voltage regulators are mounted with heat sinks however for commercial design it is recommended to incorporate fan to circulate air within the closed box [7]. In a microcontroller-based control system, software flexibility facilitates the development and updating of the control technique and uses control theory to obtain high performance.

Figure 6 has shown the input and output result in LCD display. A microcontroller can implement the controller with lower cost and smaller size than the general purpose microprocessor with accompanying external circuits, such as an A/D converter and PWM generator. The switching times of each device are implemented in software, and the PWM pulses are generated through the pulse generator of the microcontroller.

After simulation and determination of specifications of the overall design, a prototype is built of the final circuit \& the overall block diagram of the single-phase online UPS is implemented using a microcontroller PIC16C73B (Microchip) \& the operation modes and control-block diagrams of the battery charger/discharger are shown in Figure 7. In the charging mode, the dc-link voltage is regulated to be a constant voltage and the battery charger/discharger operates as a buck converter, which steps down the dc-link voltage to the battery voltage. Figure 3 shows the overall system of the UPS. It is divided into the following two parts: the controller and power circuit.

The input and output waveform \& the switching waveform of inverter switches are shown in Figure 8. The controller part included the microcontroller running the control algorithms and driver circuits. By utilizing the battery charger/discharger, the power circuit is implemented without transformer and increasing the number of the batteries. First a preliminary prototype was constructed on a breadboard to test the components before being soldered permanent on a PCB, however due to the high voltage, testing on the breadboard was limited to No load condition which makes the current to be zero. MOSFETs are attached to heat-sinks due to their nature of generating heat especially at high switching frequency.

Figure 9 has shown the digital battery charger and charge controlling with PIC12F675. Rise in temperature is brought by high frequency switching of MOSFET which dramatically decreases the power output the inverter. In order to maintain a low temperature all MOSFET and voltage regulators are mounted with heat sinks however for commercial design. It is recommended to incorporate fan to circulate air within the closed box. When one switch is $\mathrm{ON}$ at that

\section{Dral ine UPS 1 GEll}

In: 11 - 3ण D: $227 \mathrm{Y}$

Figure 6. Input and output result. 


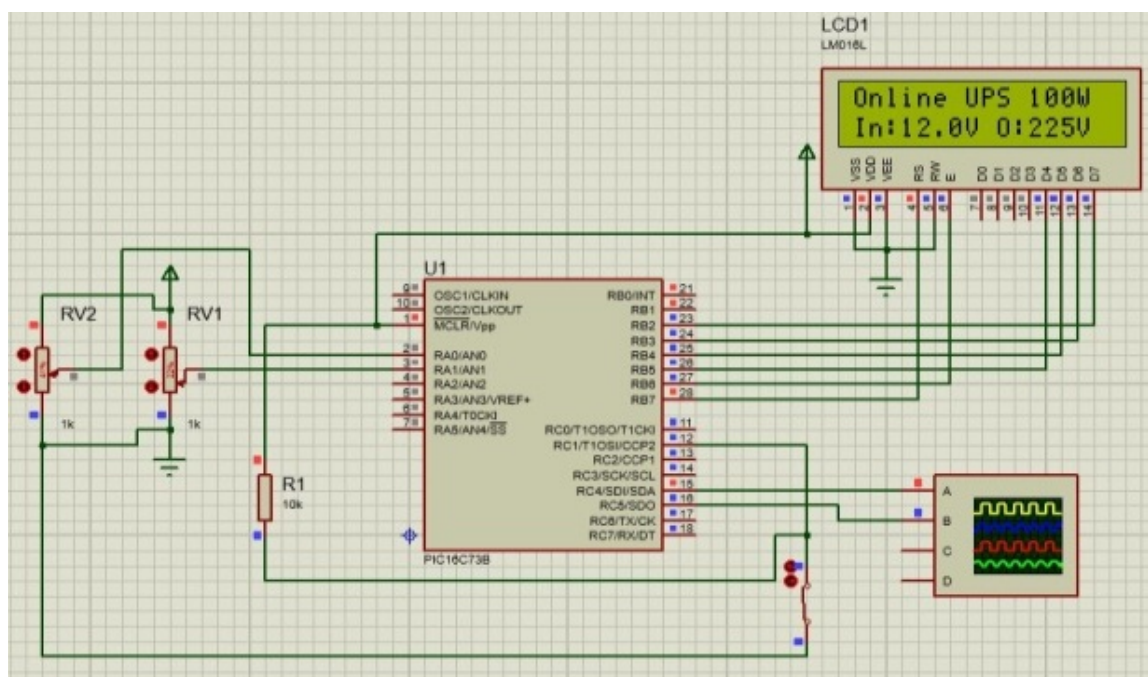

Figure 7. Simulation of inverter.

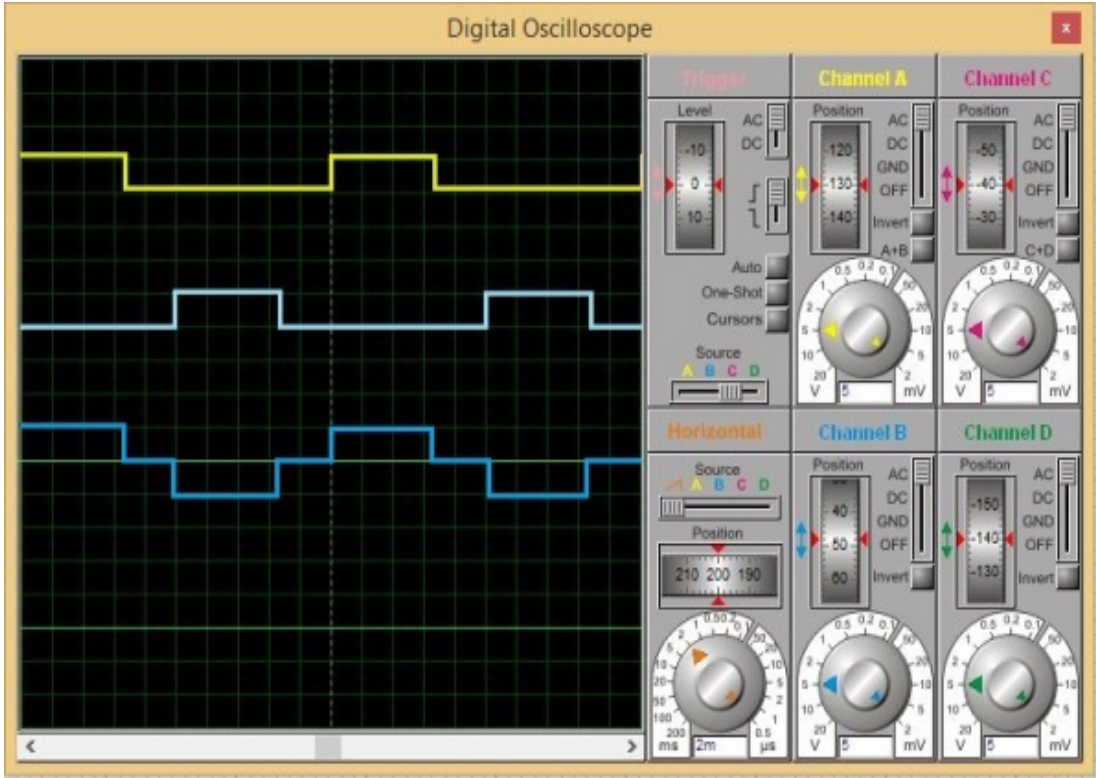

Figure 8. Digital oscilloscope.

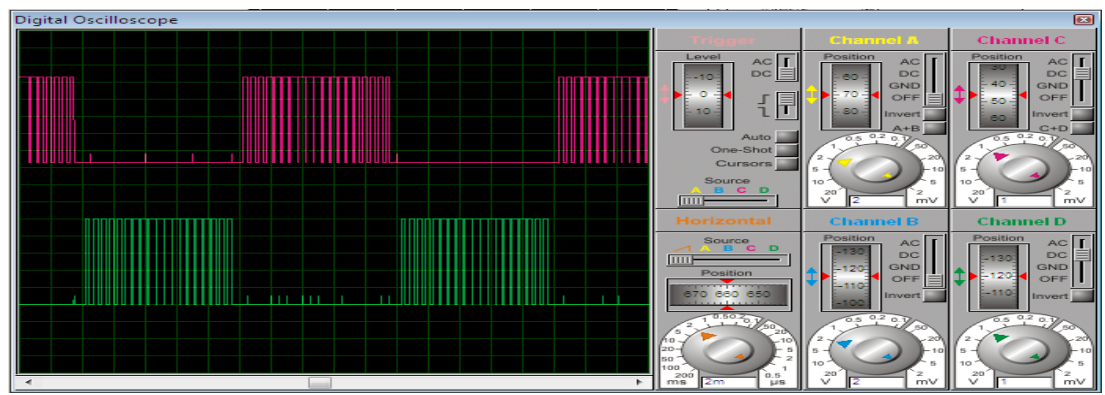

Figure 9. PWM signals generated by microcontroller.

time other switch is OFF, therefore dead band circuit is not required for push-pull arrangement of inverter. For MOSFET diver IR2110, isolated power 
supply is not required because sources of both the MOSFET switches are grounded. All individual hardware design is tested using an oscilloscope and a digital multi-meter. The key components of the overall power inverter are a PWM control circuit, a transformer, a sinusoidal PWM controller, a full-bridge inverter, and a low-pass filter. Each component was tested for the desired voltages, currents, efficiencies, and frequencies.

The above sub-sections demonstrate the results of the tests that were performed on the power inverter hardware as shown in Figure 10. The test specifications explain the methods used to show that design constraints have been met. The power inverter is composed of many components that require testing separately and as a complete system. Testing each component individually helps to locate unique problems that are specific to each component. Complete system testing will ensure that each hardware and software component is fully functional at a mutual level. When the grid power is interrupted and the system switches from grid mode to battery mode. The rectifier is no more in operation and the battery charger/discharger operates in discharging mode giving regulated dc-link voltage [8]. The transient effect in the output voltage is very small and the UPS system provides uninterruptible power to the load. The basic idea is to keep constant current charging by limiting the duty cycle of charger.

Figure 11 illustrates each main system component, the design constraints relative to each component. The solution for necessity of the qualified power for the devices that are known as critical load recently is assured with UPS. In this study for this solution the objective of the circuit was to invert power from high voltage DC sources or an output voltage of DC to DC boost into AC power similar to one available in our wall sockets for any load and of which was partially

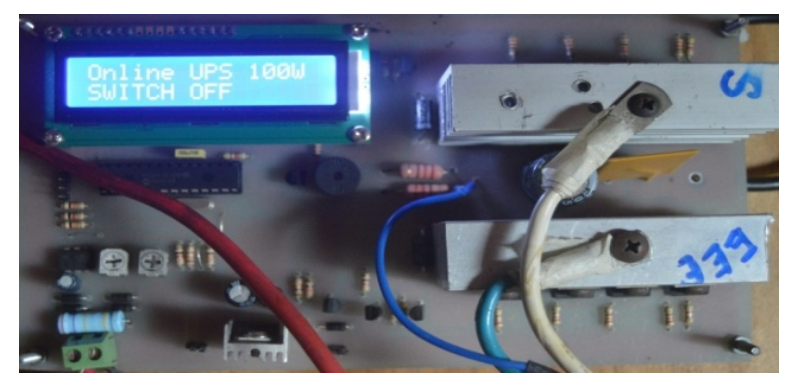

Figure 10. DSP control UPS system at power mode.

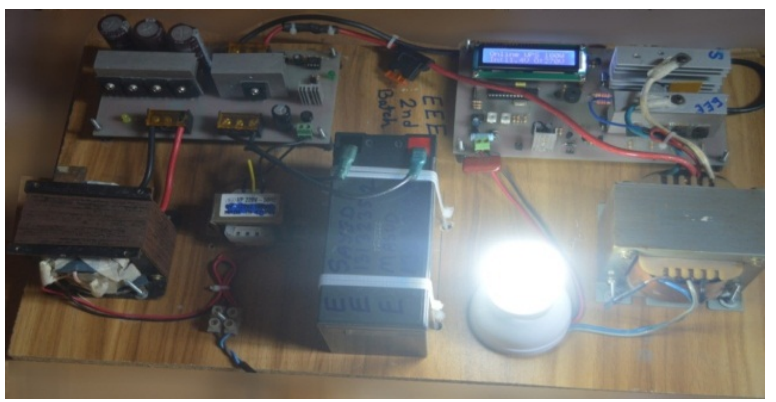

Figure 11. Completed UPS system with physical output. 
met. AC network was protected from negative effects by making input current sinusoidal as holding power factor.

\section{Conclusion}

The online UPS schemes will full-fill all the characteristics of online UPS. To ensure power supply continuity in the event of a power failure, the batteries must be charged in a good condition. The main control part of UPS (Uninterrupted Power Supply) system is traditionally designed with analog components. Due to the complicated circuit structure and difficulty in debugging, the current trend for UPS design is to adopt a microcontroller as a main control center instead of the analog solution. It can be concluded that the sole aim of carrying out the design, analysis and implementation of a smart embedded personal computer uninterrupted power supply system was achieved, in that the aim was to develop a cheap, affordable, reliable and efficient smart embedded system, which was successfully realized at the end of the design process. A well-built power protection solution, featuring high-quality, highly efficient UPS hardware, can help keep our business applications available, our power costs manageable and our data safe. By familiarizing themselves with the basics of what a UPS does and how to choose the right one for their needs, data center operators can ensure that mission-critical systems always have the clean, reliable electricity they need to drive success. There are a few changes that need to be worked on for future work. Under certain circumstances, however, double-conversion on-line can be the better choice. In particular, in geographic areas where the AC power is highly distorted and has extreme voltage variations, a double-conversion on-line UPS will go to battery less often to maintain proper output [9]. The inductor used in the filter is a transformer coil and therefore not suitable for the amount of power required. Proper inductor is recommended, iron core inductor that has small copper resistance which will increase the efficiency of the inverter [10]. Very often UPS systems require remote communication to allow for faster and more effective diagnostics during the various operating stages and quick maintenance operations. These functions may be obtained by fitting the equipment with communication boards and network interface cards and providing additional monitoring services to ensure maximum safety and peace of mind for the customer. Beside, our project is stand by modulated sine wave if we upgrade it up to fifth harmonic wave we can get better power without any kind of noise. Moreover, Batteries are key factor for the UPS system. Lead batteries can provide high current levels and operate discontinuously without necessarily reaching the end of discharge, without being affected by a "memory effect" like other types of batteries.

\section{Conflicts of Interest}

The authors declare no conflicts of interest regarding the publication of this paper. 


\section{References}

[1] Pereira, L.F.A., Vieira, J.F., Bonan, G., Coutinho, D. and da Silva, J.M.G. (2014) Multiple Resonant Controllers for Uninterruptible Power Supplies-A Systematic Robust Control Design Approach. IEEE Transactions on Industrial Electronics, 61, 1528-1538. https://doi.org/10.1109/TIE.2013.2259781

[2] Hegazy, O., Barrero, R., Van Mierlo, J., Lataire, P., Omar, N. and Coosemans, T. (2013) An Advanced Power Electronics Interface for Electric Vehicles Applications. IEEE Transactions on Power Electronics, 28, 5508-5521. https://doi.org/10.1109/TPEL.2013.2256469

[3] Gopinath, G. and Arikesh, A. (2013) Development of Three Step Charging $\mathrm{H}$-Bridge Based Sine Wave Inverter. International Journal of Advanced Trends in Computer Science and Engineering, 2, 149-153.

[4] Zhao, B., Song, Q., Liu, W. and Xiao, Y. (2013) Next-Generation Multi-Functional Modular Intelligent UPS System for Smart Grid. IEEE Transactions on Industrial Electronics, 60, 3602-3618. https://doi.org/10.1109/TIE.2012.2205356

[5] Branco, C.G., Torrico-Bascope, R.P., Cruz, C.M. and de A Lima, F. (2013) Proposal of Three-Phase High-Frequency Transformer Isolation UPS Topologies for Distributed Generation Applications. IEEE Transactions on Industrial Electronics, 60, 1520-1531. https://doi.org/10.1109/TIE.2012.2193858

[6] Bhattacharjee, A. (2012) Design and Comparative Study of Three Photovoltaic Battery Charge Control Algorithms in MATLAB/SIMULINK Environment. International Journal of Advanced Computer Research, 2, 129-135.

[7] Su, B. and Lu, Z. (2010) An Interleaved Totem-Pole Boost Bridgeless Rectifier with Reduced Reverse-Recovery Problems for Power Factor Correction. IEEE Transactions on Power Electronics, 25, 1406-1415. https://doi.org/10.1109/TPEL.2010.2040633

[8] von jouanne, A., Enjeti, P.N. and Lucas, D.J. (1996) DSP Control of High-Power UPS Systems Feeding Nonlinear Loads. IEEE Transactions on Industrial Electronics, 43, 121-125. https://doi.org/10.1109/41.481416

[9] Torrico-Bascopé, R.P., Oliveira, D., Branco, C.G. and Antunes, F.L. (2008) A UPS with $110-\mathrm{V} / 220-\mathrm{V}$ Input Voltage and High-Frequency Transformer Isolation. IEEE Transactions on Industrial Electronics, 55, 2984-2996. https://doi.org/10.1109/TIE.2008.918480

[10] Rashid, M.H. (2004) Power Electronics Circuits, Devices and Applications. 3rd Edition, Prentice-Hall of India, Private Limited, New-Delhi. 